\title{
First Quantization Coefficient Extraction from Double Compressed JPEG Images
}

\author{
Fausto Galvan ${ }^{1}$, Giovanni Puglisi ${ }^{2}$, \\ Arcangelo R. Bruna ${ }^{2}$, and Sebastiano Battiato ${ }^{2}$ \\ 1 University of Udine, Italy \\ fausto.galvan@uniud.it \\ 2 University of Catania, Italy \\ Dipartimento di Matematica e Informatica - Image Processing Lab \\ \{puglisi, bruna, battiato\}@dmi.unict.it
}

\begin{abstract}
In the forensics domain can be useful to recover image history, and in particular whether or not it has been doubly compressed. Clarify this point allows to assess if, in addition to the compression at the time of shooting, the picture was decompressed and then resaved. This is not a clear indication of forgery, but it can justify further investigations. In this paper we propose a novel technique able to retrieve the coefficients of the first compression in a double compressed JPEG image when the second compression is lighter than the first one. The proposed approach exploits the effects of successive quantizations followed by dequantization to recover the original compression parameters. Experimental results and comparisons with a state of the art method confirm the effectiveness of the proposed approach.
\end{abstract}

Keywords: Double JPEG Compression, Forgery Identification.

\section{Introduction}

The pipeline which leads to ascertain whether an image has undergone to some kind of forgery leads through the following steps: determine whether the image is "original" and, in the case where the previous step has given negative results, try to understand the past history of the image. To discover that an input image has been manipulated or not is a prelude to any other type of investigation. Regarding the preliminary stage, the EXIF metadata could be examined, but they are not so robust to tampering, so that they can provide indicative but not certain results. To discover image manipulations, many approaches have been proposed in literature. In this scenario, a lot of works as reported in [4, have proved that a very promising way is the analysis of the statistical distribution of the values assumed by the DCT coefficients. In this regard, in [11, [12] and [10] is shown how, by checking the related histogram, it is possible to determine whether the image was or not doubly saved, and also if the quantization coefficient, was greater (or less) than that used in the first compression. Other methods suggest JPEG blocking artefacts analysis [6] and the use of the hash functions [2]. In

A. Petrosino (Ed.): ICIAP 2013, Part I, LNCS 8156, pp. 783-792, 2013.

(C) Springer-Verlag Berlin Heidelberg 2013 
9], the authors suggest a method that assess whether an image has been double compressed with the same compression factor. The part of this theory not yet fully developed, at least in our knowledge, concerns the determination of the values of the coefficients of the first quantization. In [10] the authors expose some ideas to retrieve the coefficients of the first quantization: the first and the second one, just using the evaluation of the behavior of normalized histograms, provide unsatisfactory results. They then focused on a method that uses a Neural Network as a classifier. Their approach, however, does not work for medium and high frequencies, and it has been proved only for a specific subset of the AC terms. The works in [14:5] also estimate first quantization coefficients but only to locate forgeries without providing exhaustive results related to its estimation. From here on, $q_{1}$ and $q_{2}$ will indicate respectively the coefficient of first and second quantization for a generic frequency in the DCT domain. In [8] the author, to estimate $q_{1}$, proposes to carry out a third quantization and then calculates the error between the coefficients before and after this step. By varying the coefficient of the third quantization the method is able to detect two minima, one (absolute) in correspondence of $q_{2}$, and one (local) in correspondence of $q_{1}$. Due to several similarities with the proposed approach, we will discuss in detail the results of this method and its limitations in the remainder of this article.

In this paper we focused on the determination of first quantization coefficients. In particular we demonstrate that, when the second quantization factor is lower than the first one, the accuracy of the proposed approach is pretty high. This is analytically proved by exploiting some interesting properties of integer numbers whenever they are quantized more than once. The proposed method can be used as stand-alone module (just to detect forgeries) or combined with other methods (e.g., in combination with the "Signature detection" algorithm proposed in [7] to retrieve the camera model used to shoot the image). The paper is structured as follows: in Section 2.JPEG compression algorithm together with some properties of double compressed images are reviewed. Section 3 presents the mathematical details of the proposed approach whereas in Section 4 the effectiveness of the proposed solution has been tested considering both synthetic and real data (double JPEG compressed images). Finally, in Section 5 we report conclusions and our prospects for future work in this field.

\section{Scientific Background}

The JPEG compression engine 13 works just considering a partition of the input image into $8 \times 8$ non-overlapping blocks. A DCT transform is then applied to each block; next a proper dead-zone quantization is employed just using for each coefficient a corresponding integer value belonging to a $8 \times 8$ quantization matrix 3. The quantized coefficients, obtained just rounding the results of the ratio between the original DCT coefficients and the corresponding quantization values, are then transformed into a data stream by mean of a classic entropy coding. Coding parameters and other metadata are usually inserted into the header of the JPEG file to allow a proper decoding. If an image has been compressed twice 


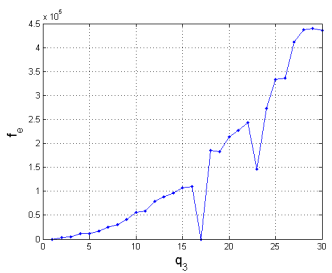

(a)

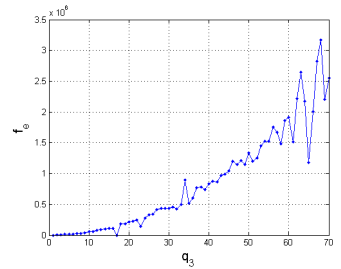

(b)

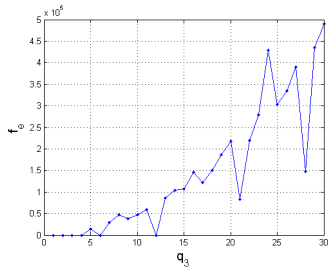

(c)

Fig. 1. Examples of the error function values (2) with respect to $q_{3}$ : (a) $q_{1}=23$, $q_{2}=17$ and $q_{3}$ is in the range $[1,30]$; (b) $q_{1}=23, q_{2}=17$ and $q_{3}$ is in the range $[1,70]$; (c) $q_{1}=21, q_{2}=12$ and $q_{3}$ is in the range $[1,30]$

(e.g., after malicious manipulation) although, of course, the current quantization values are available, the original quantization factors are lost.

Considering a single DCT coefficient $c$ and the related quantization factors $q_{1}$ (first quantization) and $q_{2}$ (second quantization), the value of each coefficient is given by $1: 2$ :

$$
\left[\left[\frac{c}{q_{1}}\right] \frac{q_{1}}{q_{2}}\right]
$$

To infer the value of $q_{1}$, Farid ( 8 ) suggests to quantize again such value with a novel quantization coefficient $\left(q_{3}\right)$ varying in a proper range and evaluate an error function defined as follow 3 :

$$
f_{e}\left(c, q_{1}, q_{2}, q_{3}\right)=\left|\left[\left[\left[\frac{c}{q_{1}}\right] \frac{q_{1}}{q_{2}}\right] \frac{q_{2}}{q_{3}}\right] q_{3}-\left[\left[\frac{c}{q_{1}}\right] \frac{q_{1}}{q_{2}}\right] q_{2}\right|
$$

For example, the typical outcome of (2) considering the DC term when $q_{1}=23$ and $q_{2}=17$ is reported in Fig. 1(a). In this specific case (both coefficients are prime numbers) we obtain interesting results: both $q_{1}$ and $q_{2}$ can be easily found, since they correspond to the two evident local minima. Hence, in this case, the first quantization coefficient $q_{1}$ can be retrieved $\left(q_{2}\right.$, as mentioned before, is already available). Unfortunately, in real cases, the original quantization coefficient cannot be easily inferred as proved by considering the following cases:

- Taking into account the quantization values used before $\left(q_{1}=23, q_{2}=17\right)$ and the same input image, but varying $q_{3}$ in the range $[1,70]$, the outcome is more complex to analyse than before. Several local minima arise and a strong one can be found in 65 (see Fig. 1(b).

\footnotetext{
${ }^{1}$ For sake of simplicity, truncation and rounding error have not been considered in (1). However, they have been taken into account in the design of the proposed approach (see Section 3)

2 [.] indicates the rounding function

3 |.|indicates the $a b s$ function
} 
- By employing the same input image and $q_{3}$ in the range $[1,30]$, but with different quantization coefficients $q_{1}=21$ and $q_{2}=12$ (they are both not prime numbers), the outcome reported in Fig, 1(c) is obtained. Without additional information about the input image, a wrong estimation could be performed $\left(q_{1}=28\right.$ in this case).

When $q_{3}=q_{2}$, the error function (2) is equal to 0 . This motivates the absolute minima found in the previous examples. Of course, the (2) allows estimating $q_{2}$, but this information is not useful since $q_{2}$ is already known from the bitstream. Obtaining a reliable $q_{1}$ estimation from (2) is a difficult task because too many cases have to be considered. In the following an alternative strategy devoted to increase the reliability of the estimation is then proposed.

\section{Proposed Approach}

As explained above, (2) allows obtaining $q_{2}$ but is not able to provide a reliable estimation of $q_{1}$. To overcome this problem we have designed a new error function:

$$
f_{e}^{\prime}\left(c, q_{1}, q_{2}, q_{3}\right)=\left|\left[\left[\left[\left[\frac{c}{q_{1}}\right] \frac{q_{1}}{q_{2}}\right] \frac{q_{2}}{q_{3}}\right] \frac{q_{3}}{q_{2}}\right] q_{2}-\left[\left[\frac{c}{q_{1}}\right] \frac{q_{1}}{q_{2}}\right] q_{2}\right|
$$

To properly understand the rationale of (3), especially when $q_{3}=q_{1}$, we have to better analyse the effect of a single quantization and dequantization step. If we examine the behaviour of the following function:

$$
\widehat{c}=\left[\frac{c}{q}\right] q
$$

we can note that if $q$ is odd, all integer numbers in $\left[n q-\left\lfloor\frac{q}{2}\right\rfloor, n q+\left\lfloor\frac{q}{2}\right\rfloor\right\rfloor$ will be mapped in $n q$ (with $n$ a generic integer number). If $q$ is even it maps in $n q$ all the integer numbers in $\left[n q-\frac{q}{2}, n q+\frac{q}{2}-1\right]$. From now on we call this range 'space attributable to $n q$ '. Such aspect is synthetically sketched in Fig. 2 (when $q$ is even). As a consequence, we can say that (4) "groups" all integer numbers of its domain in multiples of $q$. Let observe that the maximum distance between a generic coefficient $c$ and the corresponding $\widehat{c}$, obtained by the quantization and dequantization process, is $\frac{q}{2}$ if $c$ is even, $\left\lfloor\frac{q}{2}\right\rfloor$ if $c$ is odd. Based on the above observation, we analyse three rounds applied in sequence, when $q_{2}<q_{1}$ and $q_{3}=q_{1}$ :

$$
\left[\left[\left[\frac{c}{q_{1}}\right] \frac{q_{1}}{q_{2}}\right] \frac{q_{2}}{q_{1}}\right] q_{1}
$$

$\overline{4\lfloor.\rfloor \text { indicates }}$ the floor function 


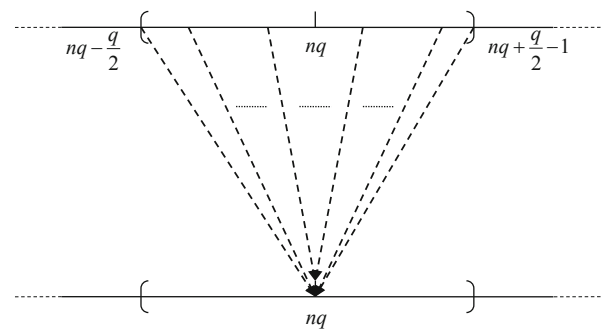

Fig. 2. The effect of quantization and dequantization for a generic term $c \in[n q-$ $\left.\frac{q}{2}, n q+\frac{q}{2}-1\right]$ in case of $q$ even

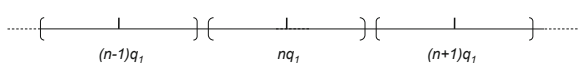

Fig. 3. The effect of quantization and dequantization for coefficient $q_{1}$

$-c_{1}=\left[\frac{c}{q_{1}}\right] q_{1}$ for the above observations leads to the situation shown in Fig. 3]

$-c_{2}=\left[\frac{c_{1}}{q_{2}}\right] q_{2}$ maps multiples of $q_{1}$ in multiples of $q_{2}$. It is worth noting that, being $q_{2}<q_{1}$, a generic $n q_{1}$ will be mapped in a multiple of $q_{2}$ (for example $m q_{2}$ ) whose distance from $n q_{1}$ will be less than or equal to $\frac{q_{2}}{2}$ (or $\left\lfloor\frac{q_{2}}{2}\right\rfloor$ if $q_{2}$ is odd), then in the space attributable to $n q_{1}$;

- at this point, $\left[\frac{c_{2}}{q_{1}}\right] q_{1}$ maps $c_{2}$ in $n q_{1}$ again, since, as pointed out in the preceding paragraph, $c_{2}$ is in the space attributable to $n q_{1}$.

With the three steps above, we demonstrated that (see Fig. 41):

$$
\left[\left[\left[\frac{c}{q_{1}}\right] \frac{q_{1}}{q_{2}}\right] \frac{q_{2}}{q_{1}}\right] q_{1}=\left[\frac{c}{q_{1}}\right] q_{1}
$$

Therefore the error function in (3) is 0 when $q_{3}=q_{1}$ regardless the $c$ value.

It is worth noting that in real conditions, due to rounding and truncation to eight bit integers, (3) in $q_{3}=q_{1}$ is close to zero but not zero. In presence of multiple minima (e.g., $q_{1}$ is a multiple of $q_{2}$ ) this behaviour has to be carefully considered as detailed below. Starting from a double quantized image $I_{D Q}$, DCT coefficient $c_{D Q}$ are extracted and, for each frequency $f_{j} \in[1,2, \ldots, 64]$, the following algorithm is applied. First, a set of candidate to be the right value of $q_{1}$ $\left(C_{f_{j}}\right)$ is collected by simply considering (3) and selecting the strongest minima with the lowest values. In order to select the correct first quantization coefficient, we exploit information coming from the double compressed image $I_{D Q}$. 


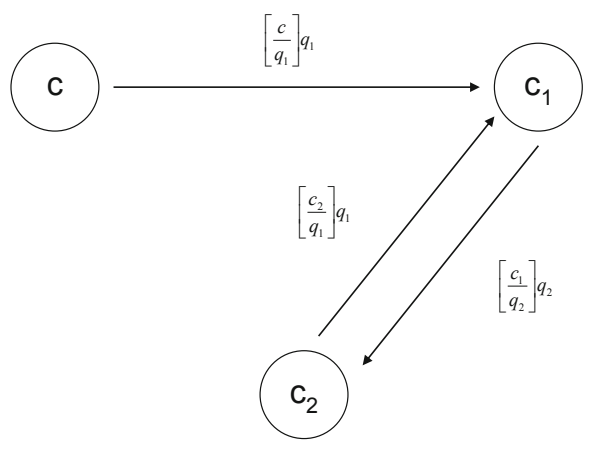

Fig. 4. Scheme describing the effect of three quantization and dequantization with coefficients $q_{1}, q_{2}$ and $q_{3}=q_{1}$ again

Specifically, as already proposed in [10, by performing a proper cropping of the double compressed image, an estimation of the original DCT coefficients can be obtained $\left(\widehat{c_{f_{j}}}\right)$. These coefficients are then used as input of a double compression procedure where the first quantization is performed by using a constant matrix with values from $C_{f_{j}}$ and the second one by simply using the already known values of the second quantization coefficients $\left(q_{2_{f_{j}}}\right.$ values are present in the header data). At the end of this step a set of double quantized images are obtained $\left(I_{D Q_{i}}, i \in\left[1, \ldots,\left|C_{f_{j}}\right|\right]\right)$ related to the different first quantization candidates. Equation (3) is then computed for each candidate image $I_{D Q_{i}}$ and the output is compared with respect to the one obtained from $I_{D Q}$ by simply using the mean absolute distance. The closest image is then found and the related $\widehat{q_{f_{j}}}$ is selected as the correct one.

\section{Experimental Results}

In order to prove the effectiveness of the proposed approach several tests and comparisons have been performed. A first test has been conducted considering artificial data. Specifically, a random vector of 5000 elements has been built by using a uniform distribution in the range [-1023,1023]. The range corresponds to an input image within the range $[0,255]$ in the spatial domain. In fact, the output of the DCT transform, as it is defined, is three bits wider of the input bit depth and it is centered in zero. These simulated DCT coefficients are then used as input of the error function we proposed (3) by considering several pairs of quantization coefficients with $q_{1}<q_{2}$. As can be easily seen from Figs. 5(a) $5(\mathrm{~b})$ and 5(c) (3) has a global minimum (equal to zero) when $q_{3}=q_{1}$. Moreover, $q_{1}$ value can be found in the range $\left.q_{3} \in\right] q_{2},+\infty[$. It is worth noting that, sometimes, more than one local minimum can be found in the range $\left.q_{3} \in\right] q_{2},+\infty[$. For example, this behaviour can arise when $q_{1}$ is a multiple of $q_{2}$ (see Fig. 5(d). However, even in this case, the correct $q_{1}$ value can be easily recovered. In fact, 


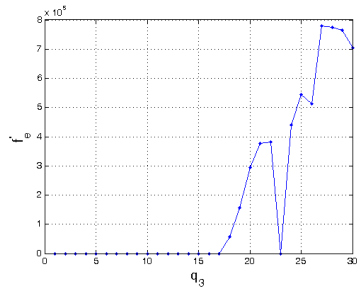

(a)

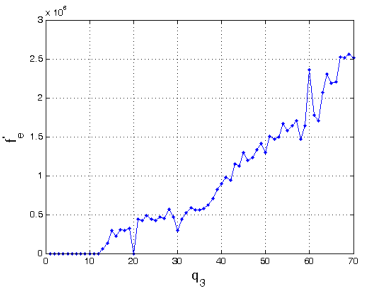

(c)

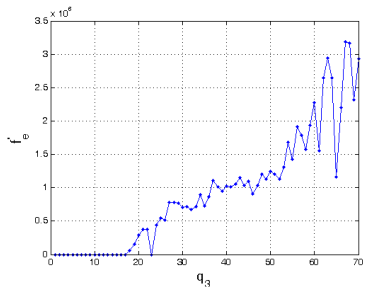

(b)

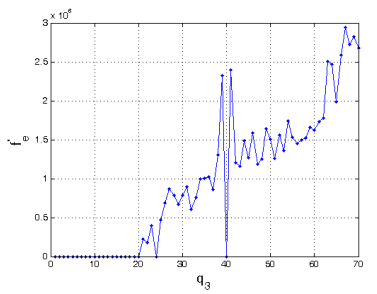

(d)

Fig. 5. Examples of error function values vs $q_{3}$, computed by using the proposed solution (3): (a) $q_{1}=23, q_{2}=17$ and $q_{3}$ is in the range [1,30]; (b) $q_{1}=23, q_{2}=17$ and $q_{3}$ is in the range [1,70]; (c) $q_{1}=20, q_{2}=12$ and $q_{3}$ is in the range [1,70]; (d) $q_{1}=40$, $q_{2}=20$ and $q_{3}$ is in the range $[1,70]$.

the additional minima are always between $q_{1}$ and $q_{2}$ and their positions depend on the common divisors between $q_{2}$ and $q_{3}$. Hence, in this case, $q_{1}$ is a multiple of $q_{2}$, the correct value is the maximum one.

To further assess the performance of our approach, several tests have been conducted considering real double compressed images. Starting from a set of 24 uncompressed images [1], by simple using JPEG encoding provided by Matlab, a dataset of double compressed images have been built just considering quality factors $\left(Q F_{1}, Q F_{2}\right)$ in the range 50 to 100 at step of 10 . Taking into account the condition $q_{1}>q_{2}$, the final dataset contains 360 images.

Tables 1, 2, 3, 4, and 5 report the average percentage of erroneously estimated $q_{1}$ values at varying of quality factor relative to the DC and the first 3, 6, 10 and 15 DCT coefficients. These values have been averaged over all images 11. The coefficients were considered in zig-zag order. This order, used in JPEG standard, allows sorting the coefficients from the lowest frequency (DC) to the highest frequencies in a $1 \mathrm{D}$ vector. As expected, better results are usually obtained for higher $Q F_{1}$ and $Q F_{2}$ values corresponding to lower quantization.

Further analyses have been conducted in order to study the performance of the proposed approach with respect to the specific DCT coefficient. In Fig. 6 is reported the average percentage of erroneously estimated $q_{1}$ values at varying of the DCT coefficient (from low to high frequencies). These values are obtained averaging over all $\left(Q F_{1}, Q F_{2}\right)$. As expected the performance of the proposed solution degrades with DCT coefficients corresponding to high frequencies. 
Table 1. Percentage of erroneously estimated $q_{1}$ values at varying of quality factor $\left(Q F_{1}, Q F_{2}\right)$ relative to the DC coefficient

\begin{tabular}{|c||c|c|c|c|c|c|}
\hline \multicolumn{1}{|c||}{} & \multicolumn{5}{c|}{$Q F_{2}$} \\
\hline \hline \multirow{5}{*}{$Q F_{1}$} & & 60 & 70 & 80 & 90 & 100 \\
\cline { 2 - 7 } & 50 & $4.17 \%$ & $0.00 \%$ & $0.00 \%$ & $0.00 \%$ & $0.00 \%$ \\
\cline { 2 - 7 } & 60 & - & $4.17 \%$ & $0.00 \%$ & $0.00 \%$ & $0.00 \%$ \\
\cline { 2 - 7 } & 70 & - & - & $0.00 \%$ & $0.00 \%$ & $0.00 \%$ \\
\cline { 2 - 7 } & 80 & - & - & - & $0.00 \%$ & $0.00 \%$ \\
\cline { 2 - 7 } & 90 & - & - & - & - & $0.00 \%$ \\
\hline
\end{tabular}

Table 2. Percentage of erroneously estimated $q_{1}$ values at varying of quality factor $\left(Q F_{1}, Q F_{2}\right)$ relative to the first 3 DCT coefficients in zig-zag order

\begin{tabular}{|c||c|c|c|c|c|c|}
\hline \multicolumn{1}{|c||}{} & \multicolumn{5}{c|}{$Q F_{2}$} \\
\hline \hline \multirow{5}{*}{$Q F_{1}$} & & 60 & 70 & 80 & 90 & 100 \\
\cline { 2 - 7 } & 50 & $1.39 \%$ & $0.00 \%$ & $0.00 \%$ & $0.00 \%$ & $0.00 \%$ \\
\cline { 2 - 7 } & 60 & - & $1.39 \%$ & $1.39 \%$ & $0.00 \%$ & $0.00 \%$ \\
\cline { 2 - 7 } & 70 & - & - & $0.00 \%$ & $0.00 \%$ & $0.00 \%$ \\
\cline { 2 - 7 } & 80 & - & - & - & $1.39 \%$ & $0.00 \%$ \\
\cline { 2 - 7 } & 90 & - & - & - & - & $0.00 \%$ \\
\hline
\end{tabular}

Table 3. Percentage of erroneously estimated $q_{1}$ values at varying of quality factor $\left(Q F_{1}, Q F_{2}\right)$ relative to the first 6 DCT coefficients in zig-zag order

\begin{tabular}{|c||c|c|c|c|c|c|}
\hline \multicolumn{1}{|c||}{} & \multicolumn{5}{c|}{$Q F_{2}$} \\
\hline \hline \multirow{5}{*}{$Q F_{1}$} & & 60 & 70 & 80 & 90 & 100 \\
\cline { 2 - 7 } & 50 & $2.08 \%$ & $0.00 \%$ & $0.00 \%$ & $0.00 \%$ & $0.00 \%$ \\
\cline { 2 - 7 } & 60 & - & $4.17 \%$ & $1.39 \%$ & $0.00 \%$ & $0.00 \%$ \\
\cline { 2 - 7 } & 70 & - & - & $0.00 \%$ & $0.00 \%$ & $0.00 \%$ \\
\cline { 2 - 7 } & 80 & - & - & - & $0.69 \%$ & $0.00 \%$ \\
\cline { 2 - 7 } & 90 & - & - & - & - & $0.00 \%$ \\
\hline
\end{tabular}

Table 4. Percentage of erroneously estimated $q_{1}$ values at varying of quality factor $\left(Q F_{1}, Q F_{2}\right)$ relative to the first $10 \mathrm{DCT}$ coefficients in zig-zag order

\begin{tabular}{|c||c|c|c|c|c|c|}
\hline \multicolumn{1}{|c||}{} & \multicolumn{6}{c|}{$Q F_{2}$} \\
\hline \hline \multirow{5}{*}{$Q F_{1}$} & & 60 & 70 & 80 & 90 & 100 \\
\cline { 2 - 7 } & 50 & $7.92 \%$ & $0.00 \%$ & $0.00 \%$ & $0.83 \%$ & $0.00 \%$ \\
\cline { 2 - 7 } & 60 & - & $10.42 \%$ & $10.42 \%$ & $0.00 \%$ & $0.00 \%$ \\
\cline { 2 - 7 } & 70 & - & - & $0.00 \%$ & $0.00 \%$ & $0.00 \%$ \\
\cline { 2 - 7 } & 80 & - & - & - & $0.42 \%$ & $0.00 \%$ \\
\cline { 2 - 7 } & 90 & - & - & - & - & $0.00 \%$ \\
\hline
\end{tabular}

To test the effectiveness of the proposed function (3), some comparisons have been performed with the same automatic algorithm described before but considering function (2) proposed in [8] instead of (3). As can be easily seen from 
Table 5. Percentage of erroneously estimated $q_{1}$ values at varying of quality factor $\left(Q F_{1}, Q F_{2}\right)$ relative to the first $15 \mathrm{DCT}$ coefficients in zig-zag order

\begin{tabular}{|c||c|c|c|c|c|c|}
\hline \multicolumn{1}{|c||}{} & \multicolumn{6}{c|}{$Q F_{2}$} \\
\hline \hline \multirow{4}{*}{$Q F_{1}$} & & 60 & 70 & 80 & 90 & 100 \\
\cline { 2 - 7 } & 50 & $19.44 \%$ & $12.78 \%$ & $0.56 \%$ & $2.22 \%$ & $5.83 \%$ \\
\cline { 2 - 7 } & 60 & - & $24.17 \%$ & $14.44 \%$ & $0.00 \%$ & $0.00 \%$ \\
\cline { 2 - 7 } & 70 & - & - & $1.67 \%$ & $0.00 \%$ & $0.00 \%$ \\
\cline { 2 - 7 } & 80 & - & - & - & $0.28 \%$ & $0.00 \%$ \\
\cline { 2 - 7 } & 90 & - & - & - & - & $0.00 \%$ \\
\hline
\end{tabular}

\section{$\longrightarrow$ Proposed Error Function (3) $\rightarrow$ Error Function used in [8]}

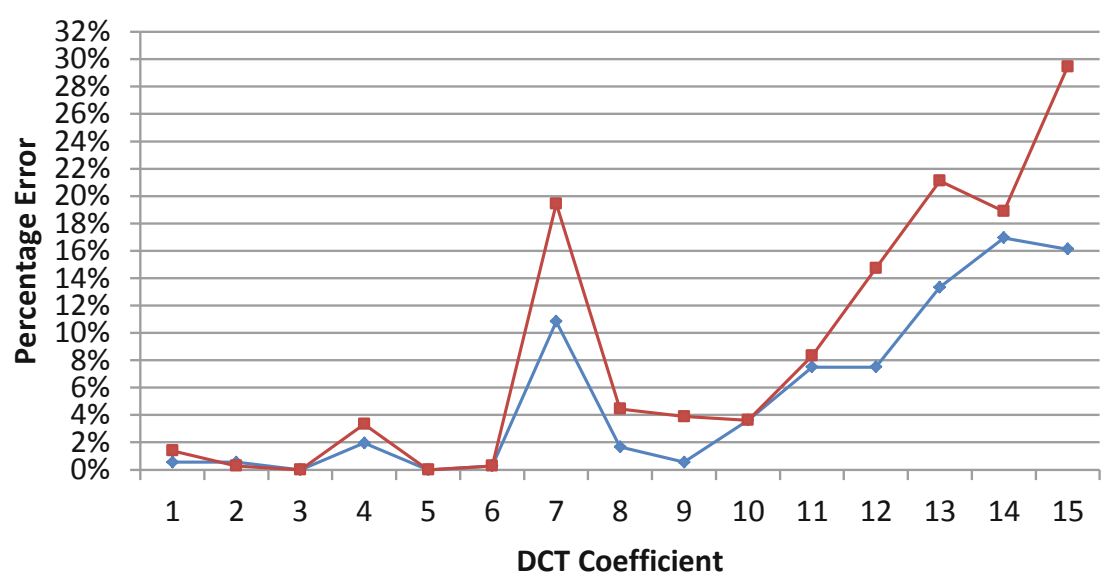

Fig. 6. Percentage of erroneously estimated $q_{1}$ values at varying of DCT coefficient position (zig-zag scanning) considering the proposed error function (3) and the one presented in 8]. These values are obtained averaging over all $\left(Q F_{1}, Q F_{2}\right)$ and images [1]

Fig. 6, the proposed approach provides satisfactory results outperforming the considered state-of-the-art approach.

\section{Conclusion}

In this paper we proposed a novel algorithm for the estimation of the first quantization coefficient from double compressed JPEG images. To confirm the effectiveness of the proposed solution, several tests have been conducted both on synthetic and real data. Comparisons have been also performed with the error function proposed in [8]. Future work will be devoted to find smart solutions able to retrieve $q_{1}$ also in the case where $q_{1} \leq q_{2}$. Moreover, additional experiments related to the recovering of the overall initial quantization matrix will be 
performed considering a double compression process achieved by applying actual quantization tables used by camera devices and common photo-retouching software (e.g., Photoshop, Gimp, etc.).

\section{References}

1. Dataset Eastman Kodak Company: PhotoCD PCD0992, http://r0k.us/graphics/kodak/

2. Battiato, S., Farinella, G.M., Messina, E., Puglisi, G.: Robust image alignment for tampering detection. IEEE Transactions on Information Forensics and Security $7(4), 1105-1117(2012)$

3. Battiato, S., Mancuso, M., Bosco, A., Guarnera, M.: Psychovisual and statistical optimization of quantization tables for DCT compression engines. In: Proceedings of the 11th International Conference on Image Analysis and Processing (ICIAP), pp. 602-606 (2001)

4. Battiato, S., Messina, G.: Digital forgery estimation into DCT domain: a critical analysis. In: Proceedings of the First ACM Workshop on Multimedia in Forensics (MiFor), pp. 37-42 (2009)

5. Bianchi, T., Piva, A.: Image forgery localization via block-grained analysis of JPEG artifacts. IEEE Transactions on Information Forensics and Security 7(3), 1003-1017 (2012)

6. Bruna, A.R., Messina, G., Battiato, S.: Crop detection through blocking artefacts analysis. In: Maino, G., Foresti, G.L. (eds.) ICIAP 2011, Part I. LNCS, vol. 6978, pp. 650-659. Springer, Heidelberg (2011)

7. Farid, H.: Digital image ballistics from JPEG quantization: A followup study. Tech. Rep. TR2008-638, Department of Computer Science, Dartmouth College (2008)

8. Farid, H.: Exposing digital forgeries from JPEG ghosts. IEEE Transactions on Information Forensics and Security 4(1), 154-160 (2009)

9. Huang, F., Huang, J., Shi, Y.Q.: Detecting double JPEG compression with the same quantization matrix. IEEE Transactions on Information Forensics and Security $5(4), 848-856(2010)$

10. Lukas, J., Fridrich, J.: Estimation of primary quantization matrix in double compressed JPEG images. In: Proceedings of Digital Forensic Research Workshop, DFRWS (2003)

11. Popescu, A., Farid, H.: Statistical tools for digital forensics. In: Fridrich, J. (ed.) IH 2004. LNCS, vol. 3200, pp. 128-147. Springer, Heidelberg (2004)

12. Redi, J., Taktak, W., Dugelay, J.: Digital image forensics: a booklet for beginners. Multimedia Tools and Applications 51(1), 133-162 (2011)

13. Wallace, G.K.: The JPEG still picture compression standard. Communications of the ACM 34(4), 30-44 (1991)

14. Wang, W., Dong, J., Tan, T.: Exploring DCT coefficient quantization effect for image tampering localization. In: Proceedings of Workshop on Information Forensics and Security (2011) 